Regenerating islet-derived family, member 4 (Reg IV), a member of the Reg gene family, has been reported to be overexpressed in gastrointestinal tract cancers. Reg IV overexpression in tumor cells has been associated with carcinogenesis, tissue regeneration, proliferation and resistance to apoptosis. Reg IV activates the epidermal growth factor receptor (EGFR) signaling pathway in colon cancer and increases expression of B-cell lymphoma-2 (Bcl-2) and B-cell lymphoma-extra large (Bcl-xl), which are associated with the inhibition of apoptosis, results in mitogenic signaling in colon cancer cells, increase cell proliferation, metastasis and decreased apoptosis. Reg IV treatment inhibits 5-fluorouracil induced apoptosis, at least two mechanisms are involved in inhibition of apoptosis by Reg IV, including Bcl-2 and dihydropyrimidine dehydrogenase (DPD). These studies may lead to novel therapeutic strategies for cancers expressing Reg IV. Recently, one proteoglycan was confirmed to disrupt this signaling pathway to perform antitumor effect. This review summaries current knowledge of the expression and roles of Reg IV in human colorectal cancer, describes the possible signaling pathway which Reg IV activates, and discusses the relevance of Reg IV as a potential therapeutic target for cancer treatment.

Key words: Reg IV, colorectal cancer, Cdx2, EGFR/Akt/AP-1 pathway, targeted therapy.

Contemp Oncol (Pozn) 2015; 19 (4): 261-264 DOI: $10.5114 /$ wo.2015.54385

\section{The role of Reg IV in colorectal cancer, as a potential therapeutic target}

\author{
Yiwang $\mathrm{Hu}^{1}$, Chi Pan ${ }^{1}$, Jiyi Hu², Suzhan Zhang ${ }^{1}$
}

${ }^{1}$ Cancer Institute (Key Laboratory of Cancer Prevention and Intervention, China National Ministry of Education), School of Medicine, Zhejiang University, Hangzhou, PR China ${ }^{2}$ Medical Oncology, The Second Affiliated Hospital, School of Medicine, Zhejiang University, Hangzhou, PR China

\section{Introduction}

Colorectal cancer is the third most commonly diagnosed cancer in males and the second in females, with over 1.2 million new cancer cases and 608,700 deaths estimated to have occurred in 2008 [1]. Despite improvements in cancer diagnosis and therapy, many patients are still diagnosed at the late stages of the disease, and the disease often recurs even after curative surgery. Multiple genetic alterations lead to carcinogenesis. Research on the candidate differentially expressed genes may help us to find biological markers for the evaluation of cancer diagnosis and offer novel molecular targets for anticancer therapy.

Over the past three decades, considerable attention has focused on the Reg (regenerating) gene family, which belongs to the calcium-dependent lectin superfamily, encoding a group of small multifunctional secretory proteins [2]. Reg family proteins are primarily involved in cell proliferation and differentiation, inflammation, diabetes, and carcinogenesis [3]. Regenerating islet-derived family member 4 (Reg IV), the most recently discovered member of the Reg gene family, is thought to be a candidate gene for cancer-specific expression [4]. In this review, we summarize current understanding of Reg IV, and discuss the relevance of Reg IV as a potential therapeutic target in the treatment of colorectal cancer.

\section{Expression and function of Reg IV in gastrointestinal tract tumors}

Reg IV is expressed in a large variety of normal tissues in humans, such as stomach, small intestine, colon and pancreas [2,5]. The expression of Reg IV is abundantly enhanced in colorectal adenocarcinoma and adenoma $[6,7]$, and gastric cancer [5, 8], whereas Reg IV expression is not detected in lung or breast cancers [5]. Therefore, Reg IV may be a good marker for gastrointestinal tumors. Previous studies have suggested that Reg IV may take part in early carcinogenesis in certain cancers. Many colorectal cancers develop through the 'adenoma to carcinoma sequence' model [9], in which adenomas are recognized as precursor lesions of the vast majority of colorectal cancers. Zhang et al. found that Reg IV was expressed in both colorectal adenoma and adenocarcinoma [7]. This result constituted further evidence that overexpression of Reg IV may be an early event in the colorectal adenoma-carcinoma sequence and carcinogenesis, and its detection may be useful in the early diagnosis of colorectal adenoma formation [10].

Whether Reg IV is associated with tumor clinicopathological features is unknown yet. Violette et al. reported that there was no significant relationship between Reg IV and the TNM state of the tumors or their localization [6]. But Yamagishi et al. suggested that Reg IV staining was observed more fre- 
quently in stage III/IV cases than in stage I/II cases, and patients with Reg IV positive gastric cancer tended to show a poor outcome, although not to a statistically significant degree [11]. In contrast to gastric carcinoma, expression of Reg IV by colorectal carcinoma is associated with lymph node metastasis. Numata et al. showed that high expression levels of Reg IV were correlated with well-differentiated histological type, deeper invasion, presence of lymphatic invasion, presence of liver metastasis, and advanced stage (stage IV) [12]. Oue et al. reported that Reg IV expression was associated with delayed liver metastasis of colorectal cancer [13], and inhibition of apoptosis by Reg IV may participate in liver metastasis. It has also been reported that Reg IV induces the expression of matrix metalloproteinase-7 (MMP-7) [14], which promotes liver metastases [15]. Overexpression of Reg IV may contribute to liver metastasis through induction of MMP-7.

\section{Transcription of the Reg IV gene may be regulated by $\mathrm{Cdx} 2$}

The caudal homeobox 2 gene $(\mathrm{Cd} \times 2)$ is reported to be involved in colorectal carcinogenesis [16] as well as the status of its differentiation [17], and is known to be a tumor suppressor [18]. The prognosis of patients with negative $\mathrm{Cd} \times 2$ expression is significantly poorer than patients with positive expression. To date, little is known about the relationship between Reg IV and Cdx2 expression. It is reported that Mucin-2 (MUC2) and Reg IV were both positive in goblet cell-like vesicles in tumor cells, and both mucin-like staining and perinuclear Reg IV staining are observed more frequently in MUC2 positive cases than in MUC2 negative cases [5]. Yamamoto et al. [19] and Werling et al. [20] found that Cdx2 interacts with the MUC2 promoter and activates MUC2 transcription. What is more, Naito et al. showed that Cdx2 protein directly regulates Reg IV expression [21]. Taken together, we suppose that $C d \times 2$ may regulate transcription of the Reg IV gene.

\section{Reg IV activates the EGFR/Akt/AP-1 signaling pathway}

Although the biological function of Reg IV is poorly understood, Reg IV is expressed in almost all epidermal growth factor receptor (EGFR) positive gastric cancers [8] and colorectal cancers $[14,22]$. Reg IV may activate EGFR and may contribute to cancer cell growth. Bishnupuri et al. found that Reg IV is a potent activator of the EGFR/Akt/ activator protein-1 (AP-1) signaling pathway in colon cancer cells and increases expression of B-cell lymphoma-2 $(\mathrm{BCl}-2)$ and $\mathrm{B}$-cell lymphoma-extra large $(\mathrm{BCl}-\mathrm{xl})$, which are proteins associated with the inhibition of apoptosis [14, 23, 24]. Activation of the EGFR signaling pathway resulted in mitogenic signaling in colon cancer cells, increased cell proliferation, angiogenesis, and metastasis, and reduced apoptosis [25]. Epidermal growth factor receptor activation indicates poor prognosis, and increases invasiveness of carcinomas and resistance to apoptotic cell death. Reg IV treatment induces tyrosine phosphorylation of the EGFR and inhibits 5-fluorouracil-induced apoptosis in gastric cancer [8].

\section{Reg IV as a therapeutic target in colorectal cancer}

It is reported that advanced colorectal adenocarcinoma is generally poorly responsive to chemotherapy and radiation $[26,27]$, and patients with Reg IV positive gastric cancer tend to show a poor outcome [28]. As demonstrated in other systems $[13,29]$, there are diverse mechanisms of resistance to drug toxicity. 5-fluorouracil (5-FU) is one of the most widely used chemotherapeutic agents for breast cancer, colorectal cancer, and gastric cancer [30]. Unfortunately, some patients show a poor response. High Reg IV expression is thought to be associated with 5-FU resistance in colon cancer cell lines [6].

\section{Possible mechanisms of Reg IV inhibition of apoptosis}

It has been reported that recombinant Reg IV increases expression of Bcl-2 [14]. B-cell lymphoma-2 is an antiapoptotic protein located on mitochondria and is expressed at high levels in some tumor cells and tissues [24, 31]. In the mitochondrial pathway, antiapoptotic Bcl-2 family proteins prevent mitochondrial membrane permeabilization and thereby inhibit changes in the mitochondrial membrane potential and cytochrome c release [31]. Mitani et al. suggested that overexpression of Reg IV may suppress 5-FU-induced apoptosis by inhibiting the mitochondrial apoptotic pathway [8].

It has been reported that AP-1 induces expression of dihydropyrimidine dehydrogenase (DPD) [32], which is an initial and rate-limiting enzyme in 5-FU catabolism, and has significance for the pharmacokinetics and toxicity of 5-FU. Overexpression of DPD in tumor cell lines is associated with resistance to 5-FU [33]. Degradation of 5-FU by induction of DPD expression may also inhibit 5-FU-induced apoptosis. Also, Mitani et al. confirmed that Reg IV inhibits apoptosis by activating the EGFR/Akt/AP-1/DPD signaling pathway, inhibiting 5-FU.

In conclusion, Reg IV can confer resistance to 5-FU-induced apoptosis in colorectal cancer and gastric cancer. At least two mechanisms are involved in inhibition of apoptosis by Reg IV, including Bcl-2 and DPD. Bishnupuri et al. reported that Reg IV is a potent activator of the EGFR/Akt/ AP-1 signaling pathway in human colon cancer cell lines [14]. Interestingly, Nanakin et al. found that EGF and TGF- $\alpha$ enhanced Reg IV gene expression by the extracellular signal-regulated kinase (ERK) signaling pathway in the SW403 cells line [22], and high phospho-ERK expression is associated with a lower survival rate of colorectal cancer [34]. These studies suggest that a positive expression feedback loop between EGF and Reg IV exists in the signaling pathways. So disruption of Reg IV/EGFR signaling may have utility as a novel therapeutic intervention for human gastrointestinal cancer.

\section{The proteoglycan ' $P 1$ ' disrupts the Reg IV/EGFR/ Akt/AP-1 signaling pathway}

$\mathrm{Li}$ et al. suggested that a proteoglycan named ' $P 1$ ' which is from Phellinus linteus (PL) could disrupt the Reg IV/EGFR/Akt/AP-1 signaling pathway [35]. They found that 


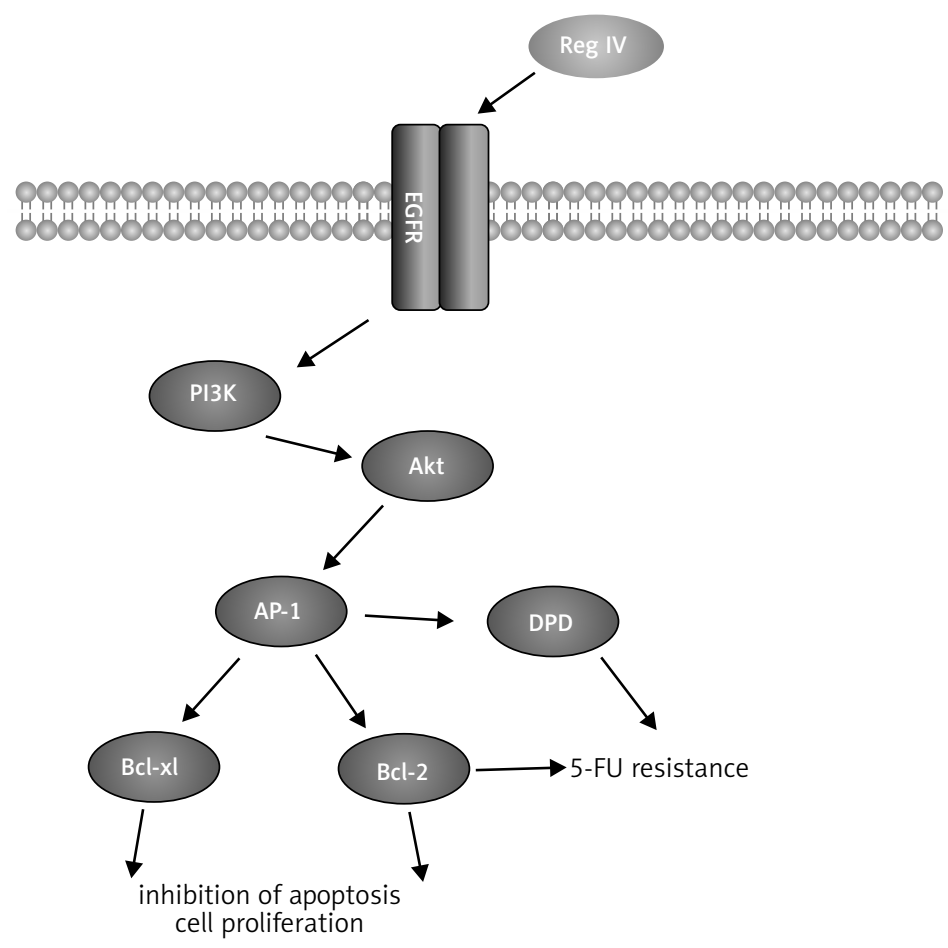

PI3K - phosphoinositide 3-kinase; AP-1 - activator protein-1; DPD - dihydropyrimidine dehydrogenase; Bcl-2 - B-cell lymphoma-2; Bcl-xl-B-cell lymphoma-extra large

Fig. 1. Reg IV activates the EGFR/Akt/AP-1 signaling pathway. Regenerating islet-derived type IV (Reg IV) could activate the epidermal growth factor receptor (EGFR) signaling pathway in colon cancer cells and increases expression of Bcl-xl and Bcl-2, resulting in cell proliferation and inhibition of apoptosis. B-cell lymphoma-2 prevents mitochondrial membrane permeabilization and leads to 5 -FU resistance. Dihydropyrimidine dehydrogenase is an initial and rate-limiting enzyme in 5-FU catabolism, which can be induced by AP-1

treatment of colonic adenocarcinoma cells with P1 resulted in significant dose-dependent inhibition in cell numbers and cell mitosis. P1 had the capacity to downregulate the expression of Reg IV and EGFR. Therefore, the proteoglycan P1 was considered to block the EGFR signaling pathway and induce Reg IV downregulation. Also, a study in vivo confirmed this hypothesis. P1 has a direct antitumor effect through inducing apoptosis and inhibiting the karyokinesis of HT-29 cells. The results are consistent with the previous report by Li et al. [36]. Therefore we expect that it will be possible to use P1 as an adjuvant chemotherapeutic and chemopreventive agent. Numerous proteins in the Reg IV/EGFR/Akt/AP-1 signaling pathway are potential therapeutic targets for colorectal cancer treatment awaiting discovery.

\section{Conclusions and future perspective}

This review focuses on the expression and roles of Reg IV in gastrointestinal tract cancers. Reg IV is generally upregulated in gastrointestinal tract cancers. Available evidence suggests that Reg IV seems to have functions, including promoting tissue regeneration, proliferation, and resistance to apoptosis, resulting in relatively worse clinicopathological features, or worse survival in patients with high Reg IV expression than those without. Reg IV is expressed in colorectal adenoma and intestinal metaplasia of the stomach, and is considered to be a good potential marker for gastrointestinal tumors. Reg IV activates the EGFR/Akt/AP-1 signaling pathway in colon cancer cells, increases cell proliferation, angiogenesis, and metastasis, and reduces apoptosis. Reducing endogenous Reg IV expression or blocking downstream signaling may be a feasible therapeutic strategy. Further investigations are still needed to confirm these observations and find more anti-cancer agents targeting the Reg IV/EGFR/Akt/AP-1 signaling pathway.

The authors declare no conflict of interest.

\section{References}

1. Jemal A, Bray F, Center MM, Ferlay J, Ward E, Forman D. Global cancer statistics. CA Cancer J Clin 2011; 61: 69-90.

2. Hartupee JC, Zhang H, Bonaldo MF, Soares MB, Dieckgraefe BK. Isolation and characterization of a cDNA encoding a novel member of the human regenerating protein family: Reg IV. Biochim Biophys Acta 2001; 1518: 287-93.

3. Zhang YW, Ding LS, Lai MD. Reg gene family and human diseases. World I Gastroenterol 2003; 9: 2635-41.

4. Aung PP, Oue N, Mitani Y, Nakayama H, Yoshida K, Noguchi T, Bosserhoff AK, Yasui W. Systematic search for gastric cancer-specific genes based on SAGE data: melanoma inhibitory activity and matrix metalloproteinase-10 are novel prognostic factors in patients with gastric cancer. Oncogene 2006; 25: 2546-57.

5. Oue N, Mitani Y, Aung PP, et al. Expression and localization of Reg IV in human neoplastic and non-neoplastic tissues: Reg IV expres- 
sion is associated with intestinal and neuroendocrine differentia tion in gastric adenocarcinoma. J Pathol 2005; 207: 185-98.

6. Violette S, Festor E, Pandrea-Vasile I, et al. Reg IV, a new member of the regenerating gene family, is overexpressed in colorectal carcinomas. Int J Cancer 2003; 103: 185-93.

7. Zhang Y, Lai M, Lv B, et al. Overexpression of Reg IV in colorectal adenoma. Cancer Lett 2003; 200: 69-76.

8. Mitani Y, Oue N, Matsumura S, et al. Reg IV is a serum biomarke for gastric cancer patients and predicts response to 5-fluorouracil-based chemotherapy. Oncogene 2007; 26: 4383-93.

9. Lin YM, Furukawa Y, Tsunoda T, Yue CT, Yang KC, Nakamura Y. Mo lecular diagnosis of colorectal tumors by expression profiles of 50 genes expressed differentially in adenomas and carcinomas. Oncogene 2002; 21: 4120-8

10. Zhang Y, Lai M, Gu X, Luo M, Shao L. Reg IV, a differentially ex pressed gene in colorectal adenoma. Chin Med J (Engl) 2003; 116 918-22.

11. Yamagishi H, Fukui H, Sekikawa A, et al. Expression profile of REG family proteins REG Ialpha and REG IV in advanced gastric cancer: comparison with mucin phenotype and prognostic markers. Mod Pathol 2009; 22: 906-13.

12. Numata M, Oshima T, Yoshihara K, et al. Relationship between RegIV gene expression to outcomes in colorectal cancer. J Surg Oncol 2011; 104: 205-9.

13. Oue N, Kuniyasu H, Noguchi T, et al. Serum concentration of Reg IV in patients with colorectal cancer: overexpression and high serum levels of Reg IV are associated with liver metastasis. Oncology 2007; 72: 371-80.

14. Bishnupuri KS, Luo Q, Murmu N, Houchen CW, Anant S, Dieckgraefe BK. Reg IV activates the epidermal growth factor receptor/Akt/ AP-1 signaling pathway in colon adenocarcinomas. Gastroenterology 2006; 130: 137-49.

15. Kioi M, Yamamoto K, Higashi S, Koshikawa N, Fujita K, Miyazaki K. Matrilysin (MMP-7) induces homotypic adhesion of human co lon cancer cells and enhances their metastatic potential in nude mouse model. Oncogene 2003; 22: 8662-70.

16. Mallo GV, Rechreche H, Frigerio JM, et al. Molecular cloning, sequencing and expression of the mRNA encoding human $C d x 1$ and $\mathrm{Cdx} 2$ homeobox. Down-regulation of $\mathrm{Cdx} 1$ and $\mathrm{Cdx} 2 \mathrm{mRNA}$ expression during colorectal carcinogenesis. Int J Cancer 1997; 74: 35-44.

17. Hinoi T, Tani M, Lucas PC, et al. Loss of CDX2 expression and mi crosatellite instability are prominent features of large cell minimally differentiated carcinomas of the colon. Am J Pathol 2001; 159: 2239-48

18. Bonhomme C, Duluc I, Martin E, et al. The Cdx2 homeobox gene has a tumour suppressor function in the distal colon in addition to a homeotic role during gut development. Gut 2003; 52: 1465-71.

19. Yamamoto $\mathrm{H}$, Bai YQ, Yuasa Y. Homeodomain protein CDX2 regulates goblet-specific MUC2 gene expression. Biochem Biophys Res Commun 2003; 300: 813-8.

20. Werling RW, Yaziji H, Bacchi CE, Gown AM. CDX2, a highly sensitive and specific marker of adenocarcinomas of intestinal origin: an immunohistochemical survey of 476 primary and metastatic carcinomas. Am J Surg Pathol 2003; 27: 303-10.

21. Naito Y, Oue N, Hinoi T, et al. Reg IV is a direct target of intestinal transcriptional factor CDX2 in gastric cancer. PLoS One 2012; 7 : e47545.

22. Nanakin A, Fukui H, Fujii S, et al. Expression of the REG IV gene in ulcerative colitis. Lab Invest 2007; 87: 304-14.

23. Bishnupuri KS, Luo Q, Sainathan SK, et al. Reg IV regulates normal intestinal and colorectal cancer cell susceptibility to radiation-induced apoptosis. Gastroenterology 2010; 138: 616-26, 626.e611612.

24. Vanderlaag K, Wang W, Fayadat-Dilman L, Wagner J, Bald L, Grein J, Janatpour MJ. Regenerating islet-derived family member, 4 mod ulates multiple receptor tyrosine kinases and mediators of drug resistance in cancer. Int J Cancer 2012; 130: 1251-63.

25. Vanhaesebroeck B, Alessi DR. The PI3K-PDK1 connection: more than just a road to PKB. Biochem J 2000; 346 Pt 3: 561-76.

26. Cunningham D. Current status of colorectal cancer: CPT-11 (irinotecan), a therapeutic innovation. Eur J Cancer 1996; 32A Suppl 3: S1-8.
27. Cunningham D, Humblet Y, Siena S, et al. Cetuximab monotherapy and cetuximab plus irinotecan in irinotecan-refractory metastatic colorectal cancer. N Engl J Med 2004; 351: 337-45.

28. Zheng HC, Sugawara A, Okamoto H, Takasawa S, Takahashi H, Masuda S, Takano Y. Expression profile of the REG gene family in colorectal carcinoma. J Histochem Cytochem 2011; 59: 106-15.

29. Sekikawa A, Fukui H, Fujii S, et al. REG Ialpha protein may function as a trophic and/or anti-apoptotic factor in the development of gastric cancer. Gastroenterology 2005; 128: 642-53.

30. Longley DB, Harkin DP, Johnston PG. 5-fluorouracil: mechanisms of action and clinical strategies. Nat Rev Cancer 2003; 3: 330-8.

31. Vander Heiden MG, Thompson CB. BCl-2 proteins: regulators of apoptosis or of mitochondrial homeostasis? Nat Cell Biol 1999; 1 E209-16.

32. Ukon K, Tanimoto K, Shimokuni T, et al. Activator protein accelerates dihydropyrimidine dehydrogenase gene transcription in cancer cells. Cancer Res 2005; 65: 1055-62.

33. Takebe N, Zhao SC, Ural AU, Johnson MR, Banerjee D, Diasio RB, Bertino JR. Retroviral transduction of human dihydropyrimidine dehydrogenase cDNA confers resistance to 5-fluorouracil in murine hematopoietic progenitor cells and human CD34+-enriched peripheral blood progenitor cells. Cancer Gene Ther 2001; 8: 966 73.

34. Tai CJ, Chang CC, Jiang MC, et al. Clinical-pathological correlation of K-Ras mutation and ERK phosphorylation in colorectal cancer. Pol J Pathol 2012; 63: 93-100

35. Li YG, Ji DF, Zhong S, Zhu JX, Chen S, Hu GY. Anti-tumor effects of proteoglycan from Phellinus linteus by immunomodulating and inhibiting Reg IV/EGFR/Akt signaling pathway in colorectal carcinoma. Int J Biol Macromol 2011; 48: 511-7.

36. Li G, Kim DH, Kim TD, et al. Protein-bound polysaccharide from Phellinus linteus induces G2/M phase arrest and apoptosis in SW480 human colon cancer cells. Cancer Lett 2004; 216: 175-81.

\section{Address for correspondence}

\section{Suzhan Zhang}

Cancer Institute (Key Laboratory

of Cancer Prevention and Intervention,

China National Ministry of Education)

School of Medicine, Zhejiang University

Hangzhou, 310000, People's Republic of China

e-mail: Zhangscy07@gmail.com

Submitted: 11.09 .2013

Accepted: 22.11.2013 